\title{
New Traveling Wave Solutions by the Extended Generalized Riccati Equation Mapping Method of the $(2+1)$-Dimensional Evolution Equation
}

\author{
Hasibun Naher and Farah Aini Abdullah \\ School of Mathematical Sciences, Universiti Sains Malaysia (USM), 11800 Penang, Malaysia \\ Correspondence should be addressed to Hasibun Naher, hasibun06tasauf@gmail.com \\ Received 19 September 2012; Accepted 14 October 2012 \\ Academic Editor: Mohamed A. Abdou
}

Copyright (c) 2012 H. Naher and F. A. Abdullah. This is an open access article distributed under the Creative Commons Attribution License, which permits unrestricted use, distribution, and reproduction in any medium, provided the original work is properly cited.

The generalized Riccati equation mapping is extended with the basic $\left(G^{\prime} / G\right)$-expansion method which is powerful and straightforward mathematical tool for solving nonlinear partial differential equations. In this paper, we construct twenty-seven traveling wave solutions for the (2+1)-dimensional modified Zakharov-Kuznetsov equation by applying this method. Further, the auxiliary equation $G^{\prime}(\eta)=w+u G(\eta)+v G^{2}(\eta)$ is executed with arbitrary constant coefficients and called the generalized Riccati equation. The obtained solutions including solitons and periodic solutions are illustrated through the hyperbolic functions, the trigonometric functions, and the rational functions. In addition, it is worth declaring that one of our solutions is identical for special case with already established result which verifies our other solutions. Moreover, some of obtained solutions are depicted in the figures with the aid of Maple.

\section{Introduction}

The study of analytical solutions for nonlinear partial differential equations (PDEs) has become more imperative and stimulating research fields in mathematical physics, engineering sciences, and other technical arena [1-47]. In the recent past, a wide range of methods have been developed to construct traveling wave solutions of nonlinear PDEs such as, the inverse scattering method [1], the Backlund transformation method [2], the Hirota bilinear transformation method [3], the bifurcation method [4, 5], the Jacobi elliptic function expansion method [6-8], the Weierstrass elliptic function method [9], the direct algebraic method [10], the homotopy perturbation method [11, 12], the Exp-function method [13-17], and others [18-28].

Recently, Wang et al. [29] presented a widely used method, called the ( $\left.G^{\prime} / G\right)$-expansion method to obtain traveling wave solutions for some nonlinear evolution equations (NLEEs). Further, in this method, the second-order linear ordinary differential equation 
$G^{\prime \prime}(\eta)+\lambda G^{\prime}(\eta)+\mu G(\eta)=0$ is implemented, as an auxiliary equation, where $\lambda$ and $\mu$ are constant coefficients. Afterwards, many researchers investigated many nonlinear PDEs to construct traveling wave solutions via this powerful $\left(G^{\prime} / G\right)$-expansion method. For example, Feng et al. [30] applied the same method for obtaining exact solutions of the KolmogorovPetrovskii-Piskunov equation. In [31], Naher et al. concerned about this method to construct traveling wave solutions for the higher-order Caudrey-Dodd-Gibbon equation. Zayed and Al-Joudi [32] studied some nonlinear partial differential equations to obtain analytical solutions by using the same method whereas Gepreel [33] executed this method and found exact solutions of nonlinear PDEs with variable coefficients in mathematical physics. Abazari and Abazari [34] constructed exact solutions for the Hirota-Ramani equation by using this method. Ozis and Aslan [35] established some traveling wave solutions for the Kawahara type equations via the same method. Naher et al. [36] investigated higher dimensional nonlinear evolution equation for obtaining traveling wave solutions by applying the improved $\left(G^{\prime} / G\right)$-expansion method. Naher and Abdullah [37] implemented this method to construct some new traveling wave solutions of the nonlinear reaction diffusion equation whilst they [38] studied the combined KdV-MKdV equation for obtaining abundant solutions via the same method. And [39] they executed this method to establish some new traveling wave solutions of the $(2+1)$-dimensional modified Zakharov-Kuznetsov equation and so on.

Zhu [40] investigated the $(2+1)$-dimensional Boiti-Leon-Pempinelle equation by applying the generalized Riccati equation mapping with the extended tanh-function method. In addition, $G^{\prime}(\eta)=w+u G(\eta)+v G^{2}(\eta)$ is used, as an auxiliary equation and called generalized Riccati equation, where $u, v$, and $w$ are arbitrary constants. Bekir and Cevikel [41] concerned about the tanh-coth method combined with the Riccati equation to study nonlinear coupled equation in mathematical physics. Guo et al. [42] implemented the extended Riccati equation mapping method for solving the diffusion-reaction and the $\mathrm{mKdV}$ equation with variable coefficient whilst Li et al. [43] studied higher-dimensional Jimbo-Miwa equation via the generalized Riccati equation expansion method. Salas [44] obtained some exact solutions for the Caudrey-Dodd-Gibbon equation by applying the projective Riccati equation method whereas Naher and Abdullah [45] studied the modified Benjamin-Bona-Mahony equation via the generalized Riccati equation mapping with the basic $\left(G^{\prime} / G\right)$-expansion method for constructing traveling wave solutions and so on.

Many researchers implemented various methods to investigate the (2+1)-dimensional modified Zakharov-Kuznetsov equation. For instance, Khalfallah [46] used homogeneous balance method to establish traveling wave solutions of this equation. In [47], Bekir applied basic $\left(G^{\prime} / G\right)$-expansion method for obtaining exact traveling wave solutions for the same equation. In this basic $\left(G^{\prime} / G\right)$-expansion method, they employed second-order linear ordinary differential equation (LODE) with constant coefficients, as an auxiliary equation instead of generalized Riccati equation.

The importance of our present work is, in order to construct many new traveling wave solutions including solitons, periodic, and rational solutions, a $(2+1)$-dimensional Modified Zakharov-Kuznetsov equation considered by applying the extended generalized Riccati equation mapping method.

\section{The Extended Generalized Riccati Equation Mapping Method}

Suppose the general nonlinear partial differential equation

$$
H\left(v, v_{t}, v_{x}, v_{y}, v_{x t}, v_{y t}, v_{x y}, v_{t t}, v_{x x}, v_{y y}, \ldots\right)=0
$$


where $v=v(x, y, t)$ is an unknown function, $H$ is a polynomial in $v(x, y, t)$, and the subscripts indicate the partial derivatives.

The most important steps of the generalized Riccati equation mapping together with the $\left(G^{\prime} / G\right)$-expansion method $[29,40]$ are as follows.

Step 1. Consider the traveling wave variable:

$$
v(x, y, t)=g(\eta), \quad \eta=x+y-C t
$$

where $C$ is the speed of the traveling wave. Now using (2.2), (2.1) is converted into an ordinary differential equation for $g(\eta)$ :

$$
F\left(g, g^{\prime}, g^{\prime \prime}, g^{\prime \prime \prime}, \ldots\right)=0
$$

where the superscripts stand for the ordinary derivatives with respect to $\eta$.

Step 2. Equation (2.3) integrates term by term one or more times according to possibility and yields constant(s) of integration. The integral constant(s) may be zero for simplicity.

Step 3. Suppose that the traveling wave solution of (2.3) can be expressed in the form $[29,40]$

$$
g(\eta)=\sum_{j=0}^{n} e_{j}\left(\frac{G^{\prime}}{G}\right)^{j}
$$

where $e_{j}(j=0,1,2, \ldots, n)$ and $e_{n} \neq 0$, with $G=G(\eta)$ is the solution of the generalized Riccati equation:

$$
G^{\prime}=w+u G+v G^{2}
$$

where $u, v, w$ are arbitrary constants and $v \neq 0$.

Step 4 . To decide the positive integer $n$, consider the homogeneous balance between the nonlinear terms and the highest order derivatives appearing in (2.3).

Step 5. Substitute (2.4) along with (2.5) into the (2.3), then collect all the coefficients with the same order, the left hand side of (2.3) converts into polynomials in $G^{k}(\eta)$ and $G^{-k}(\eta),(k=$ $0,1,2, \ldots)$. Then equating each coefficient of the polynomials to zero and yield a set of algebraic equations for $e_{j}(j=0,1,2, \ldots, n), u, v, w$, and $C$.

Step 6. Solve the system of algebraic equations which are found in Step 5 with the aid of algebraic software Maple to obtain values for $e_{j}(j=0,1,2, \ldots, n)$ and $C$ then, substitute obtained values in (2.4) along with (2.5) with the value of $n$, we obtain exact solutions of (2.1). (2.5).

In the following, we have twenty seven solutions including four different families of 
Family 1. When $u^{2}-4 v w>0$ and $u v \neq 0$ or $v w \neq 0$, the solutions of (2.5) are:

$$
\begin{aligned}
& G_{1}=\frac{-1}{2 v}\left(u+\sqrt{u^{2}-4 v w} \tanh \left(\frac{\sqrt{u^{2}-4 v w}}{2} \eta\right)\right), \\
& G_{2}=\frac{-1}{2 v}\left(u+\sqrt{u^{2}-4 v w} \operatorname{coth}\left(\frac{\sqrt{u^{2}-4 v w}}{2} \eta\right)\right), \\
& G_{3}=\frac{-1}{2 v}\left(u+\sqrt{u^{2}-4 v w}\left(\tanh \left(\sqrt{u^{2}-4 v w} \eta\right) \pm \operatorname{sech}\left(\sqrt{u^{2}-4 v w} \eta\right)\right),\right. \\
& G_{4}=\frac{-1}{2 v}\left(u+\sqrt{u^{2}-4 v w}\left(\operatorname{coth}\left(\sqrt{u^{2}-4 v w} \eta\right) \pm \operatorname{csch}\left(\sqrt{u^{2}-4 v w \eta}\right)\right)\right), \\
& G_{5}=\frac{-1}{4 v}\left(2 u+\sqrt{u^{2}-4 v w}\left(\tanh \left(\frac{\sqrt{u^{2}-4 v w}}{4} \eta\right)+\operatorname{coth}\left(\frac{\sqrt{u^{2}-4 v w}}{4} \eta\right)\right),\right. \\
& G_{6}=\frac{1}{2 v}\left(-u+\frac{ \pm \sqrt{\left(D^{2}+E^{2}\right)\left(u^{2}-4 v w\right)}-D \sqrt{u^{2}-4 v w} \cosh \left(\sqrt{u^{2}-4 v w} \eta\right)}{D \sinh \left(\sqrt{u^{2}-4 v w} \eta\right)+E}\right), \\
& G_{7}=\frac{1}{2 v}\left(-u-\frac{ \pm \sqrt{\left(D^{2}+E^{2}\right)\left(u^{2}-4 v w\right)}+D \sqrt{u^{2}-4 v w} \cosh \left(\sqrt{u^{2}-4 v w \eta}\right)}{D \sinh \left(\sqrt{u^{2}-4 v w} \eta\right)+E}\right),
\end{aligned}
$$

where $D$ and $E$ are two nonzero real constants.

$$
\begin{aligned}
& G_{8}=\frac{2 w \cosh \left(\left(\sqrt{u^{2}-4 v w} / 2\right) \eta\right)}{\sqrt{u^{2}-4 v w} \sinh \left(\left(\sqrt{u^{2}-4 v w} / 2\right) \eta\right)-u \cosh \left(\left(\sqrt{u^{2}-4 v w} / 2\right) \eta\right)} \\
& G_{9}=\frac{-2 w \sinh \left(\left(\sqrt{u^{2}-4 v w} / 2\right) \eta\right)}{u \sinh \left(\left(\sqrt{u^{2}-4 v w} / 2\right) \eta\right)-\sqrt{u^{2}-4 v w} \cosh \left(\left(\sqrt{u^{2}-4 v w} / 2\right) \eta\right)} \\
& G_{10}=\frac{2 w \cosh \left(\sqrt{u^{2}-4 v w} \eta\right)}{\sqrt{u^{2}-4 v w} \sinh \left(\sqrt{u^{2}-4 v w} \eta\right)-u \cosh \left(\sqrt{u^{2}-4 v w} \eta\right) \pm i \sqrt{u^{2}-4 v w}} \\
& G_{11}=\frac{2 w \sinh \left(\sqrt{u^{2}-4 v w} \eta\right)}{-u \sinh \left(\sqrt{u^{2}-4 v w} \eta\right)+\sqrt{u^{2}-4 v w} \cosh \left(\sqrt{u^{2}-4 v w} \eta\right) \pm \sqrt{u^{2}-4 v w}} \\
& G_{12}=\frac{4 w \sinh \left(\left(\sqrt{u^{2}-4 v w} / 4\right) \eta\right) \cosh \left(\left(\sqrt{u^{2}-4 v w} / 4\right) \eta\right)}{-2 u \sinh \left(\left(\sqrt{u^{2}-4 v w} / 4\right) \eta\right) \cosh \left(\left(\sqrt{u^{2}-4 v w} / 4\right) \eta\right)+\Delta_{1}}
\end{aligned}
$$

where $\Delta_{1}=2 \sqrt{u^{2}-4 v w} \cosh ^{2}\left(\left(\sqrt{u^{2}-4 v w} / 4\right) \eta\right)-\sqrt{u^{2}-4 v w}$. 
Family 2. When $u^{2}-4 v w<0$ and $u v \neq 0$ or $v w \neq 0$, the solutions of (2.5) are:

$$
\begin{aligned}
& G_{13}=\frac{1}{2 v}\left(-u+\sqrt{4 v w-u^{2}} \tan \left(\frac{\sqrt{4 v w-u^{2}}}{2} \eta\right)\right) \\
& G_{14}=\frac{-1}{2 v}\left(u+\sqrt{4 v w-u^{2}} \cot \left(\frac{\sqrt{4 v w-u^{2}}}{2} \eta\right)\right) \\
& G_{15}=\frac{1}{2 v}\left(-u+\sqrt{4 v w-u^{2}}\left(\tan \left(\sqrt{4 v w-u^{2}} \eta\right) \pm \sec \left(\sqrt{4 v w-u^{2}} \eta\right)\right)\right) \\
& G_{16}=\frac{-1}{2 v}\left(u+\sqrt{4 v w-u^{2}}\left(\cot \left(\sqrt{4 v w-u^{2}} \eta\right) \pm \csc \left(\sqrt{4 v w-u^{2}} \eta\right)\right)\right) \\
& G_{17}=\frac{1}{4 v}\left(-2 u+\sqrt{4 v w-u^{2}}\left(\tan \left(\frac{\sqrt{4 v w-u^{2}}}{4} \eta\right)-\cot \left(\frac{\sqrt{4 v w-u^{2}}}{4} \eta\right)\right)\right. \\
& G_{18}=\frac{1}{2 v}\left(-u+\frac{ \pm \sqrt{\left(D^{2}-E^{2}\right)\left(4 v w-u^{2}\right)}-D \sqrt{4 v w-u^{2}} \cos \left(\sqrt{4 v w-u^{2}} \eta\right)}{D \sin \left(\sqrt{4 v w-u^{2}} \eta\right)+E}\right) \\
& G_{19}=\frac{1}{2 v}\left(-u-\frac{ \pm \sqrt{\left(D^{2}-E^{2}\right)\left(4 v w-u^{2}\right)}+D \sqrt{4 v w-u^{2}} \cos \left(\sqrt{4 v w-u^{2}} \eta\right)}{D \sin \left(\sqrt{4 v w-u^{2}} \eta\right)+E}\right)
\end{aligned}
$$

where $D$ and $E$ are two nonzero real constants and satisfy $D^{2}-E^{2}>0$.

$$
\begin{aligned}
& G_{20}=\frac{-2 w \cos \left(\left(\sqrt{4 v w-u^{2}} / 2\right) \eta\right)}{\sqrt{4 v w-u^{2}} \sin \left(\left(\sqrt{4 v w-u^{2}} / 2\right) \eta\right)+u \cos \left(\left(\sqrt{4 v w-u^{2}} / 2\right) \eta\right)}, \\
& G_{21}=\frac{2 w \sin \left(\left(\sqrt{4 v w-u^{2}} / 2\right) \eta\right)}{-u \sin \left(\left(\sqrt{4 v w-u^{2}} / 2\right) \eta\right)+\sqrt{4 v w-u^{2}} \cos \left(\left(\sqrt{4 v w-u^{2}} / 2\right) \eta\right)}, \\
& G_{22}=\frac{-2 w \cos \left(\sqrt{4 v w-u^{2}} \eta\right)}{\sqrt{4 v w-u^{2}} \sin \left(\sqrt{4 v w-u^{2}} \eta\right)+u \cos \left(\sqrt{4 v w-u^{2}} \eta\right) \pm \sqrt{4 v w-u^{2}}}, \\
& G_{23}=\frac{2 w \sin \left(\sqrt{4 v w-u^{2}} \eta\right)}{-u \sin \left(\sqrt{4 v w-u^{2}} \eta\right)+\sqrt{4 v w-u^{2}} \cos \left(\sqrt{4 v w-u^{2}} \eta\right) \pm \sqrt{4 v w-u^{2}}} \\
& G_{24}=\frac{4 w \sin \left(\left(\sqrt{4 v w-u^{2}} / 4\right) \eta\right) \cos \left(\left(\sqrt{4 v w-u^{2}} / 4\right) \eta\right)}{-2 u \sin \left(\left(\sqrt{4 v w-u^{2}} / 4\right) \eta\right) \cos \left(\left(\sqrt{4 v w-u^{2}} / 4\right) \eta\right)+\Delta_{2}}
\end{aligned}
$$

where $\Delta_{2}=2 \sqrt{4 v w-u^{2}} \cos ^{2}\left(\left(\sqrt{4 v w-u^{2}} / 4\right) \eta\right)-\sqrt{4 v w-u^{2}}$. 
Family 3. When $w=0$ and $u v \neq 0$, the solution (2.5) becomes:

$$
\begin{aligned}
G_{25} & =\frac{-u f_{1}}{v\left(f_{1}+\cosh (u \eta)-\sinh (u \eta)\right)}, \\
G_{26} & =\frac{-u(\cosh (u \eta)+\sinh (u \eta))}{v\left(f_{1}+\cosh (u \eta)+\sinh (u \eta)\right)},
\end{aligned}
$$

where $f_{1}$ is an arbitrary constant.

Family 4. when $v \neq 0$ and $w=u=0$, the solution of (2.5) becomes:

$$
G_{27}=\frac{-1}{v \eta+l_{1}}
$$

where $l_{1}$ is an arbitrary constant.

\section{Applications of the Method}

In this section, we have constructed new traveling wave solutions for the $(2+1)$-dimensional modified Zakharov-Kuznetsov equation by using the method.

\subsection{The (2+1)-Dimensional Modified Zakharov-Kuznetsov Equation}

We consider the $(2+1)$-dimensional Modified Zakharov-Kuznetsov equation followed by Bekir [47]

$$
u_{t}+u^{2} u_{x}+u_{x x x}+u_{x y y}=0
$$

Now, we use the wave transformation (2.2) into the (3.1), which yields:

$$
-C g^{\prime}+u^{2} g^{\prime}+2 g^{\prime \prime \prime}=0
$$

Equation (3.2) is integrable, therefore, integrating with respect $\eta$ once yields:

$$
Q-C g+\frac{1}{3} g^{3}+2 g^{\prime \prime}=0
$$

where $Q$ is an integral constant which is to be determined later.

Taking the homogeneous balance between $g^{3}$ and $g^{\prime \prime}$ in (3.3), we obtain $n=1$.

Therefore, the solution of (3.3) is of the form:

$$
g(\eta)=e_{1}\left(\frac{G^{\prime}}{G}\right)+e_{0}, \quad e_{1} \neq 0
$$


Using (2.5), (3.4) can be rewritten as

$$
g(\eta)=e_{1}\left(u+w G^{-1}+v G\right)+e_{0}
$$

where $u, v$, and $w$ are free parameters.

By substituting (3.5) into (3.3), the left hand side is converted into polynomials in $G^{k}$ and $G^{-k}(k=0,1,2, \ldots)$. Setting each coefficient of these resulted polynomials to zero, we obtain a set of algebraic equations for $e_{0}, e_{1}, u, v, w, Q$, and $C$ (algebraic equations are not shown, for simplicity). Solving the system of algebraic equations with the help of algebraic software Maple, we obtain

$$
e_{0}=\mp u i \sqrt{3}, \quad e_{1}= \pm 2 i \sqrt{3}, \quad C=-u^{2}-8 v w, \quad Q=8 u v w i \sqrt{3} \text {. }
$$

Family 5. The soliton and soliton-like solutions of (3.1) (when $u^{2}-4 v w>0$ and $u v \neq 0$ or $v w \neq 0)$ are:

$$
g_{1}= \pm 2 i \sqrt{3} \frac{2 \Psi^{2} \operatorname{sech}^{2}(\Psi \eta)}{u+2 \Psi \tanh (\Psi \eta)} \mp u i \sqrt{3}
$$

where $\Psi=(1 / 2) \sqrt{u^{2}-4 v w}, \eta=x+y+\left(u^{2}+8 v w\right) t$ and $u, v, w$ are arbitrary constants.

$$
\begin{gathered}
g_{2}=\mp 2 i \sqrt{3} \frac{2 \Psi^{2} \operatorname{csch}^{2}(\Psi \eta)}{u+2 \Psi \operatorname{coth}(\Psi \eta)} \mp u i \sqrt{3}, \\
g_{3}= \pm 2 i \sqrt{3} \frac{4 \Psi^{2} \operatorname{sech}(2 \Psi \eta)(1 \mp i \sinh (2 \Psi \eta))}{u \cosh (2 \Psi \eta)+2 \Psi \sinh (2 \Psi \eta) \pm i 2 \Psi} \mp u i \sqrt{3} \\
g_{4}=\mp 2 i \sqrt{3} \frac{2 \Psi^{2} \operatorname{csch}(\Psi \eta)}{u \sinh (\Psi \eta)+2 \Psi \cosh (\Psi \eta)} \mp u i \sqrt{3} \\
g_{6}=\mp 2 i \sqrt{3} \frac{4 D \Psi^{2}\left(D-E \sinh (2 \Psi \eta)-\sqrt{\left(D^{2}+E^{2}\right)} \cosh (2 \Psi \eta)\right)}{(D \sinh (2 \Psi \eta)+E) \Omega_{1}} \mp u i \sqrt{3}, \\
g_{7}=\mp 2 i \sqrt{3} \frac{4 \Psi^{2} \operatorname{csch}(2 \Psi \eta)}{u \tanh (\Psi \eta)+2 \Psi} \mp u i \sqrt{3} \\
(D \sinh (2 \Psi \eta)+E) \Omega_{2}
\end{gathered}
$$


where $\Omega_{1}=u D \sinh (2 \Psi \eta)+u E-2 \Psi \sqrt{\left(D^{2}+E^{2}\right)}+2 D \Psi \cosh (2 \Psi \eta), \Omega_{2}=u D \sinh (2 \Psi \eta)+u E+$ $2 \Psi \sqrt{\left(D^{2}+E^{2}\right)}+2 D \Psi \cosh (2 \Psi \eta), D$ and $E$ are two nonzero real constants.

$$
\begin{gathered}
g_{8}=\mp 2 i \sqrt{3} \frac{2 \Psi^{2} \operatorname{sech}(\Psi \eta)}{2 \Psi \sinh (\Psi \eta)-u \cosh (\Psi \eta)} \mp u i \sqrt{3}, \\
g_{9}= \pm 2 i \sqrt{3} \frac{2 \Psi^{2} \operatorname{csch}(\Psi \eta)}{2 \Psi \cosh (\Psi \eta)-u \sinh (\Psi \eta)} \mp u i \sqrt{3} \\
g_{10}= \pm 2 i \sqrt{3} \frac{4 \Psi^{2} \operatorname{sech}(2 \Psi \eta)(1 \mp i \sinh (2 \Psi \eta))}{u \cosh (2 \Psi \eta)-2 \Psi \sinh (2 \Psi \eta) \mp i 2 \Psi} \mp u i \sqrt{3} \\
g_{11}= \pm 2 i \sqrt{3} \frac{4 \Psi^{2} \operatorname{csch}(2 \Psi \eta)(1 \pm \cosh (2 \Psi \eta))}{2 \Psi \cosh (2 \Psi \eta)-u \sinh (2 \Psi \eta) \pm 2 \Psi} \mp u i \sqrt{3} \\
g_{12}= \pm 2 i \sqrt{3} \frac{2 \Psi^{2} \operatorname{csch}(\Psi \eta)}{2 \Psi \cosh (\Psi \eta)-u \sinh (\Psi \eta)} \mp u i \sqrt{3} .
\end{gathered}
$$

Family 6. The periodic form solutions of (3.1) (when $u^{2}-4 v w<0$ and $u v \neq 0$ or $v w \neq 0$ ) are:

$$
g_{13}= \pm 2 i \sqrt{3} \frac{2 \Theta^{2} \sec ^{2}(\Theta \eta)}{-u+2 \Theta \tan (\Theta \eta)} \mp u i \sqrt{3}
$$

where $\Theta=(1 / 2) \sqrt{4 v w-u^{2}}, \eta=x+y+\left(u^{2}+8 v w\right) t$ and $u, v, w$ are arbitrary constants.

$$
\begin{gathered}
g_{14}=\mp 2 i \sqrt{3} \frac{2 \Theta^{2} \csc ^{2}(\Theta \eta)}{u+2 \Theta \cot (\Theta \eta)} \mp u i \sqrt{3}, \\
g_{15}= \pm 2 i \sqrt{3} \frac{4 \Theta^{2} \sec (2 \Theta \eta)(1 \pm \sin (2 \Theta \eta))}{-u \cos (2 \Theta \eta)+2 \Theta \sin (2 \Theta \eta) \pm 2 \Theta} \mp u i \sqrt{3} \\
g_{16}=\mp 2 i \sqrt{3} \frac{2 \Theta^{2} \sec (\Theta \eta)}{u \cos (\Theta \eta)+2 \Theta \sin (\Theta \eta)} \mp u i \sqrt{3}, \\
g_{18}=\mp 2 i \sqrt{3} \frac{4 D \Theta^{2}\left(\sqrt{\left(D^{2}-E^{2}\right)} \cos (2 \Theta \eta)-E \sin (2 \Theta \eta)-D\right)}{(D \sin (2 \Theta \eta)+E) \Omega_{3}} \mp u i \sqrt{3} . \\
g_{19}=\mp 2 i \sqrt{3} \frac{4 D \Theta^{2}\left(\sqrt{\left(D^{2}-E^{2}\right)} \cos (2 \Theta \eta)+E \sin (2 \Theta \eta)+D\right)}{(D \sin (2 \Theta \eta)+E) \Omega_{4}} \mp u i \sqrt{3},
\end{gathered}
$$




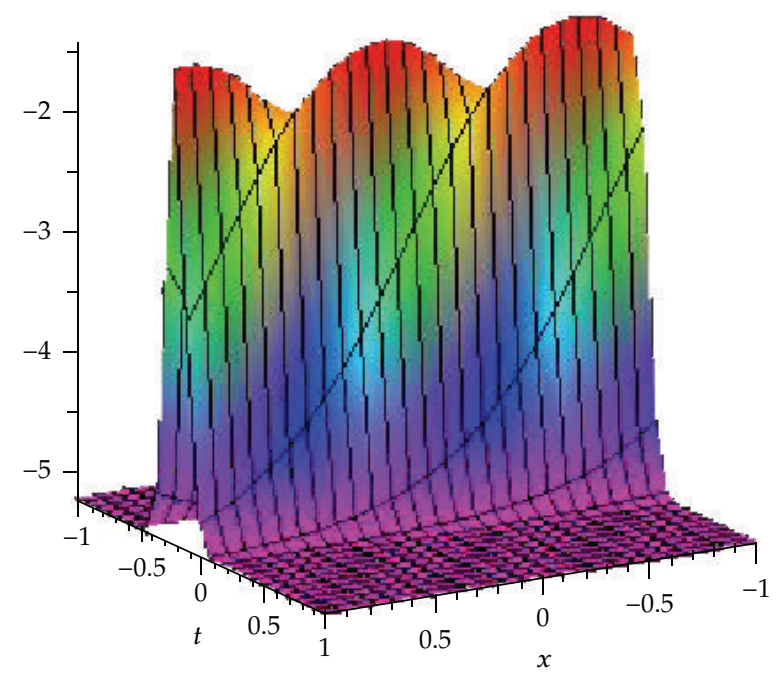

Figure 1: Solitons solution for $u=3, v=0.5, w=0.25$.

where $\Omega_{3}=u D \sin (2 \Theta \eta)+2 D \Theta \cos (2 \Theta \eta)+u E-2 \Theta \sqrt{\left(D^{2}-E^{2}\right)}, \Omega_{4}=u D \sin (2 \Theta \eta)+2 D \Theta$ $\cos (2 \Theta \eta)+u E+2 \Theta \sqrt{\left(D^{2}-E^{2}\right)}, D$ and $E$ are two nonzero real constants and satisfies $D^{2}-E^{2}>$ 0 .

$$
\begin{gathered}
g_{20}=\mp 2 i \sqrt{3} \frac{4 \Theta^{2} \csc (2 \Theta \eta)}{u \cot (\Theta \eta)+2 \Theta} \mp u i \sqrt{3}, \\
g_{21}=\mp 2 i \sqrt{3} \frac{4 \Theta^{2} \csc (2 \Theta \eta)}{u \tan (\Theta \eta)+2 \Theta} \mp u i \sqrt{3}, \\
g_{22}=\mp 2 i \sqrt{3} \frac{2 \Theta^{2} \sec (2 \Theta \eta)(1 \pm \sin (2 \Theta \eta))(u \cos (2 \Theta \eta)+2 \Theta \sin (2 \Theta \eta) \pm 2 \Theta)}{\left(u^{2}-2 v w\right) \cos ^{2}(2 \Theta \eta)+2 \Theta(1 \pm \sin (2 \Theta \eta))(2 \Theta \pm u \cos (2 \Theta \eta))} \mp u i \sqrt{3}, \\
g_{23}= \pm 2 i \sqrt{3} \frac{2 \Theta^{2} \csc (2 \Theta \eta)(-u \sin (2 \Theta \eta)+2 \Theta \cos (2 \Theta \eta) \pm 2 \Theta)}{\left(2 v w-u^{2}\right) \cos (2 \Theta \eta)-2 u \Theta \sin (2 \Theta \eta) \pm 2 v w} \mp u i \sqrt{3}, \\
g_{24}=\mp 2 i \sqrt{3} \frac{2 \Theta^{2} \csc (\Theta \eta)}{u \sin (\Theta \eta)+2 \Theta \cos (\Theta \eta)} \mp u i \sqrt{3} .
\end{gathered}
$$

Family 7. The soliton and soliton-like solutions of (3.1) (when $w=0$ and $u v \neq 0$ ) are:

$$
\begin{aligned}
& g_{25}= \pm 2 i \sqrt{3} \frac{u(\cosh (u \eta)-\sinh (u \eta))}{f_{1}+\cosh (u \eta)-\sinh (u \eta)} \mp u i \sqrt{3} \\
& g_{26}= \pm 2 i \sqrt{3} \frac{u f_{1}}{f_{1}+\cosh (u \eta)+\sinh (u \eta)} \mp u i \sqrt{3}
\end{aligned}
$$

where $f_{1}$ is an arbitrary constant, $\eta=x+y+\left(u^{2}+8 v w\right) t$. 


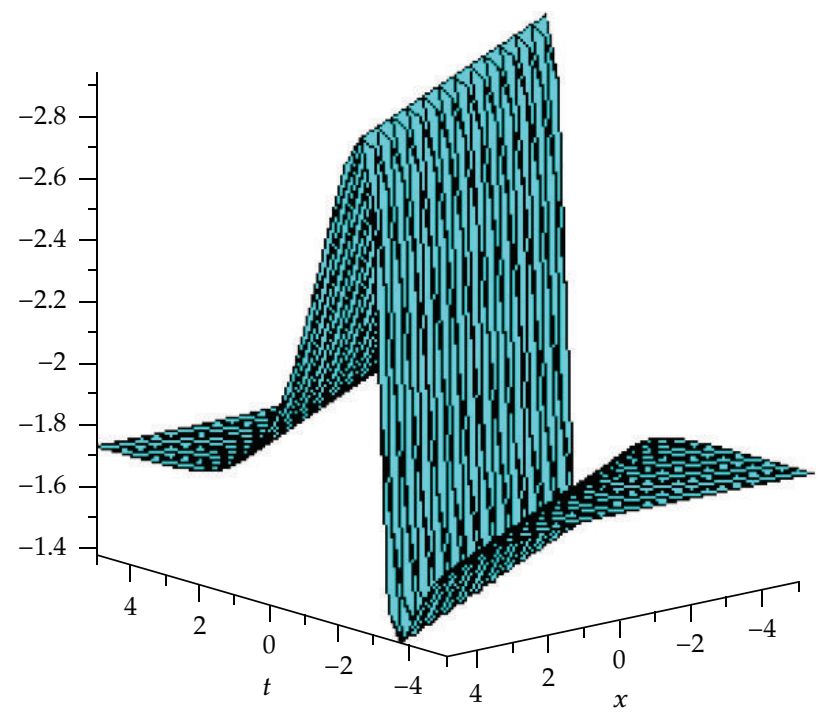

Figure 2: Periodic solution for $u=1, v=1, w=0.125$.

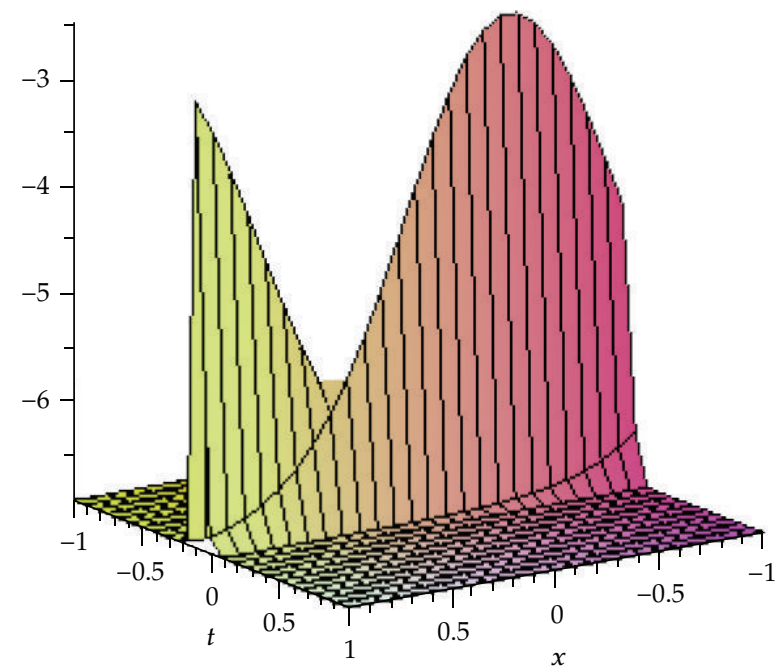

Figure 3: Solitons solution for $u=4, v=0.5, w=1$.

Family 8. The rational function solution (when $v \neq 0$ and $w=u=0$ ) is:

$$
g_{27}=\frac{\mp 2 i v \sqrt{3}}{v \eta+l_{1}}
$$

where $l_{1}$ is an arbitrary constant and $\eta=x+y+\left(u^{2}+8 v w\right) t$. 


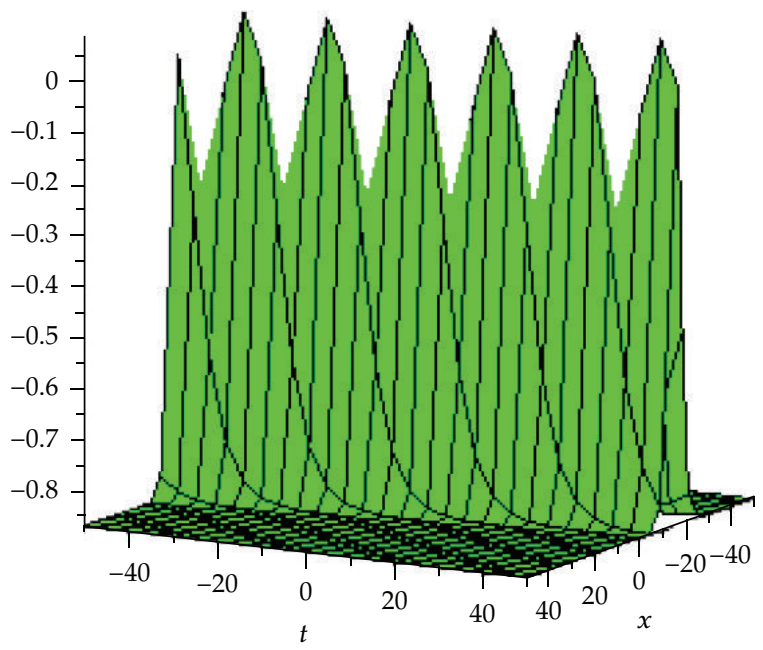

Figure 4: Solitons solution for $u=0.5, v=25.10^{-6}, w=25.10^{-5}$.

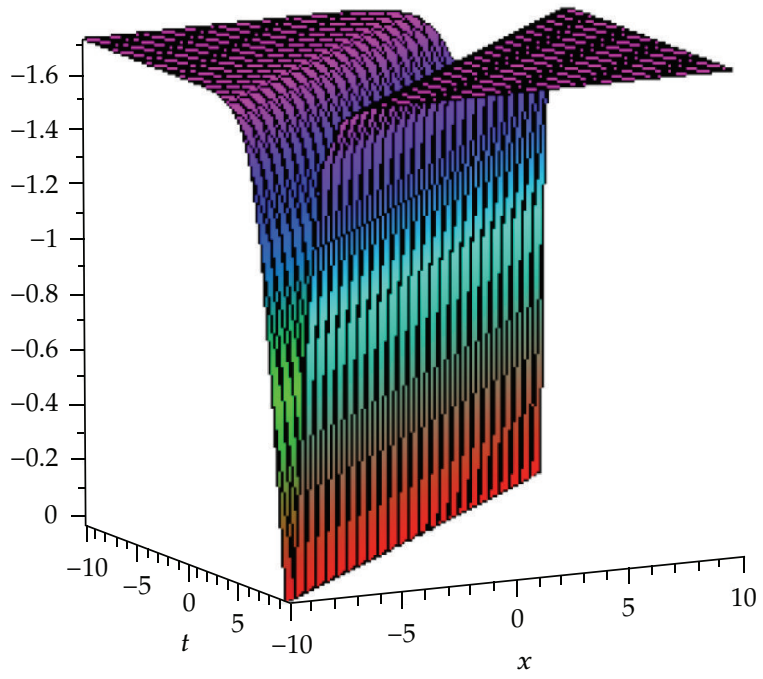

Figure 5: Periodic solution for $u=1, v=25.10^{-5}, w=25.10^{-3}$.

\section{Results and Discussion}

It is significant to mention that one of our solutions is coincided for some special case with already published results which are presented in Table 1. Furthermore, some of newly constructed solutions are illustrated in Figures 1, 2, 3, 4, 5, 6, 7, 8, 9, 10, 11, and 12. 
Table 1: Comparison between Bekir [47] solutions and newly obtained solutions.

\begin{tabular}{ll}
\hline Bekir [47] solutions & New solutions \\
\hline (i) If $C_{1}=1, C_{2}=\frac{1}{2}$ and $\lambda^{2}-4 \mu=0$, solution & (i) If $v=\frac{1}{2}, l_{1}=1, y=0$ and $g_{27}(\eta)=u_{5,6}(\xi)$, \\
Equation $(4.9)$ (from Section 4 ) becomes: & solution $g_{27}$ becomes: \\
$u_{5,6}(\xi)= \pm i \sqrt{3} \frac{2}{2+x}$. & $u_{5,6}(\xi)= \pm i \sqrt{3} \frac{2}{2+x}$. \\
\hline (ii) $C_{1}=1, C_{2}=\frac{-1}{2}$ and $\lambda^{2}-4 \mu=0$, solution & (ii) If $v=\frac{-1}{2}, l_{1}=1, y=0$ and $g_{27}(\eta)=u_{5,6}(\xi)$, \\
Equation $(4.9)$ (from Section 4 ) becomes: & solution $g_{27}$ becomes: \\
$u_{5,6}(\xi)=\mp i \sqrt{3} \frac{2}{2-x}$. & $u_{5,6}(\xi)=\mp i \sqrt{3} \frac{2}{2-x}$. \\
\hline (iii) $C_{1}=0, C_{2}=1$ and $\lambda^{2}-4 \mu=0$, solution & (iii) If $v=1, l_{1}=0, y=0$ and $g_{27}(\eta)=u_{5,6}(\xi)$, \\
Equation $(4.9)($ from Section 4$)$ becomes: & solution $g_{27}$ becomes: \\
$u_{5,6}(\xi)=\mp i \sqrt{3}\left(\frac{2}{x}\right)$. & $u_{5,6}(\xi)=\mp i \sqrt{3}\left(\frac{2}{x}\right)$. \\
\hline
\end{tabular}



Figure 6: Solitons solution for $u=1, v=0.45, w=3$.

As in Table 1, we have newly constructed traveling wave solutions $g_{1}$ to $g_{26}$ which are not being stated in the earlier literature.

\subsection{Graphical Depictions of Newly Obtained Traveling Wave Solutions}

The graphical descriptions of some solutions are represented in Figures 1-12 with the aid of commercial software Maple. 


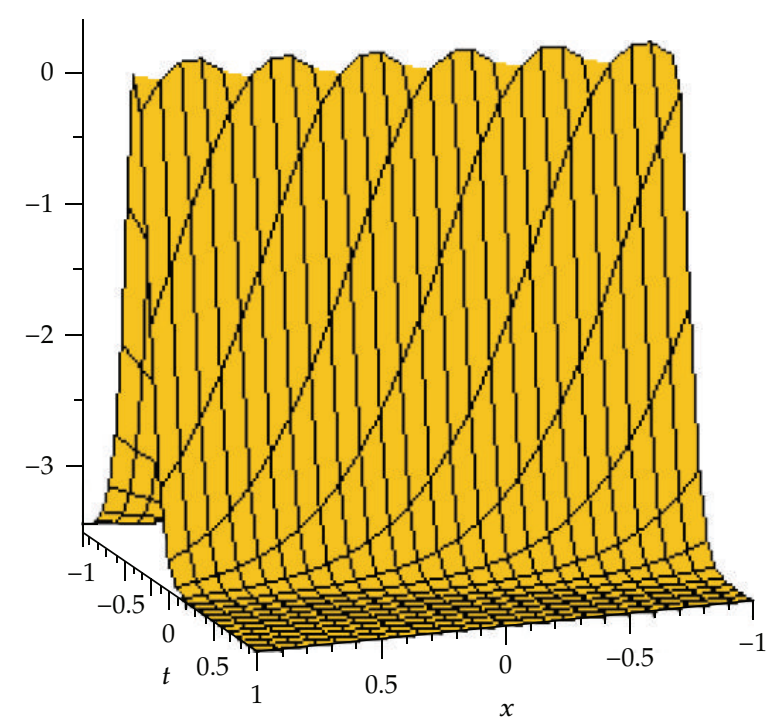

Figure 7: Solitons solution for $u=2, v=25.10^{-4}, w=25.10^{-3}$.

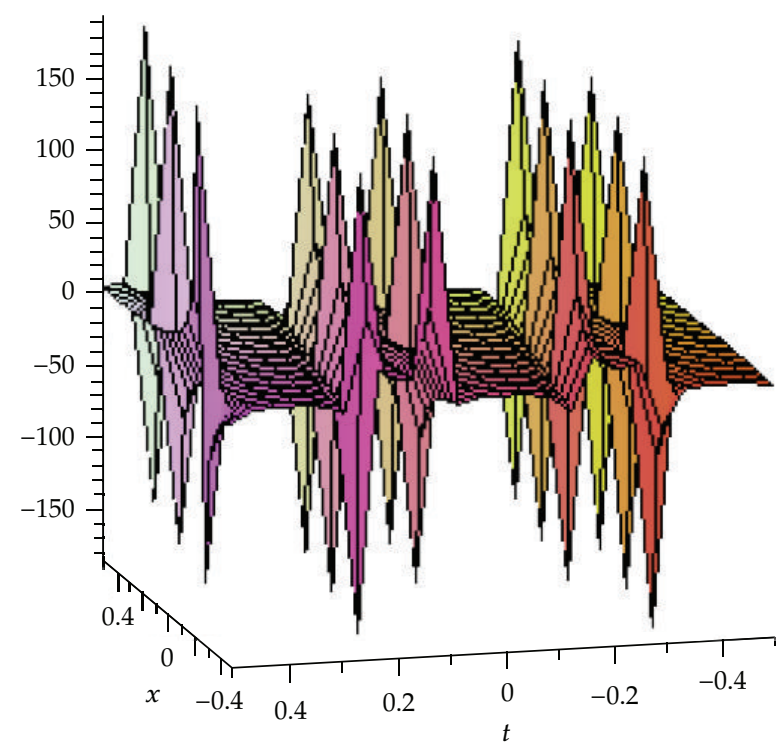

Figure 8: Solitons solution for $u=1, v=1, w=1$.

\section{Conclusions}

In this paper, we have investigated the $(2+1)$-dimensional modified Zakharov-Kuznetsov equation via the extended generalized Riccati equation mapping method. Twenty seven exact traveling wave solutions are constructed including solitons and periodic wave solutions by applying this powerful method. In addition, newly obtained solutions are depicted in terms of the hyperbolic, the trigonometric, and the rational functional form. The obtained solutions 


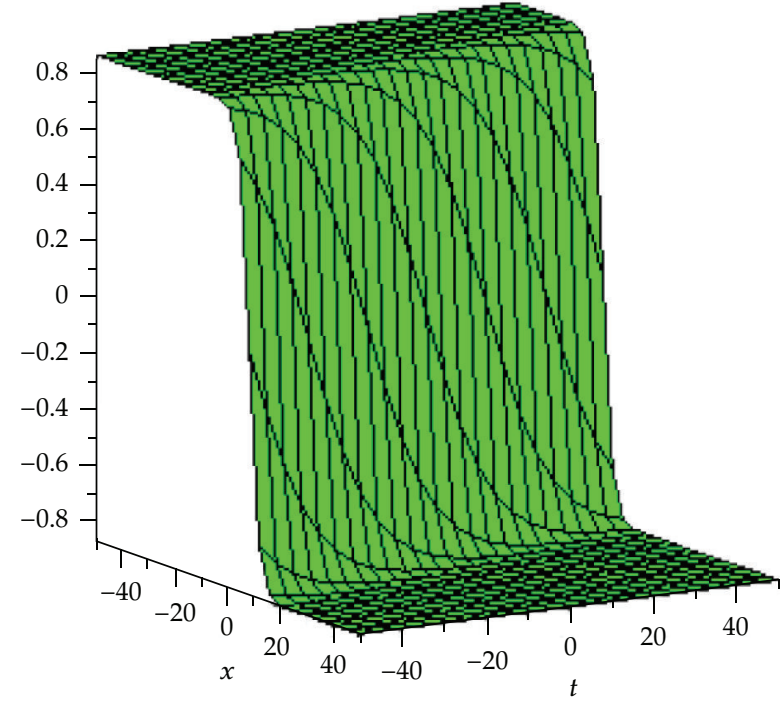

Figure 9: Periodic solution for $u=0.5, v=7, w=0, f_{1}=0.125$.



Figure 10: Solitons solution for $u=1, v=0.5, w=1$.

reveal that this method is a promising mathematical tool because it can establish a variety of new solutions of dissimilar physical structures if compared with existing methods. The correctness of newly constructed solutions is verified to be compared with already published results. Consequently, nonlinear evolution equations which regularly arise in many scientific real-time application fields can be studied by applying the extended generalized Riccati equation mapping method. 


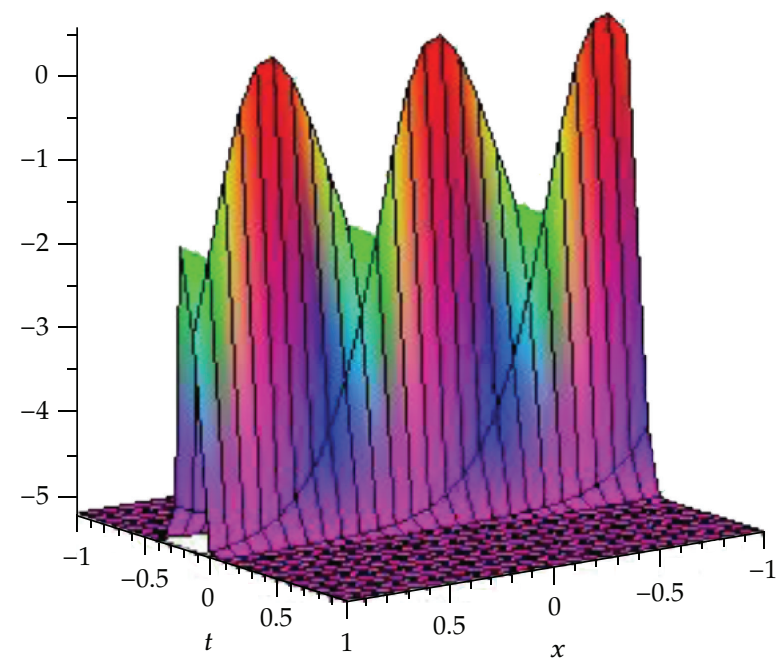

Figure 11: Solitons solution for $u=3, v=25.10^{-4}, w=25 \cdot 10^{-3}$.

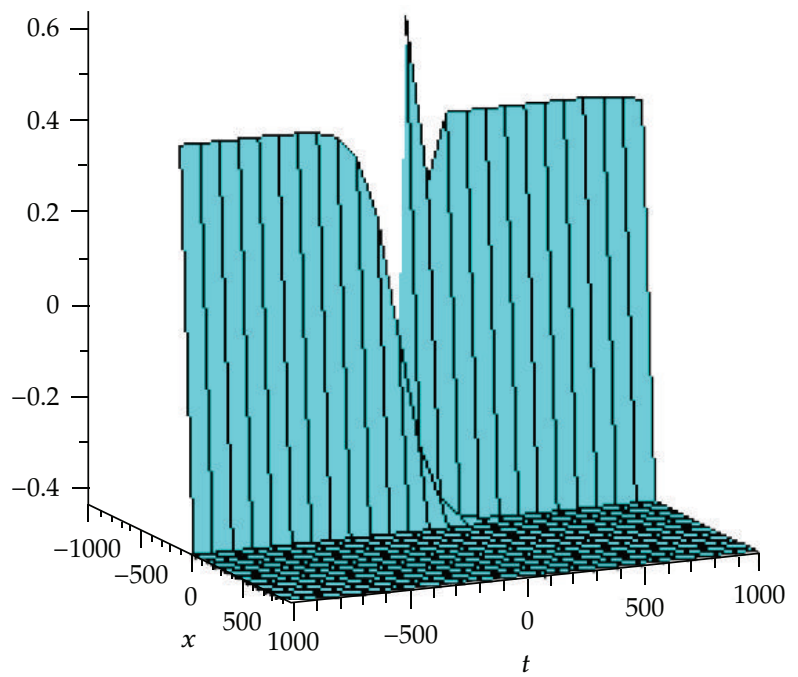

Figure 12: Periodic solution for $u=0.25, v=-5, w=0, f_{1}=0.25$.

\section{Acknowledgments}

This paper is supported by the USM short-term Grant (Ref. no. 304/PMATHS/6310072), and the authors would like to express their thanks to the School of Mathematical Sciences, USM for providing related research facilities.

\section{References}

[1] M. J. Ablowitz and P. A. Clarkson, Solitons, Nonlinear Evolution Equations and Inverse Scattering, Cambridge University Press, Cambridge, UK, 1991. 
[2] C. Rogers and W. F. Shadwick, Backlund Transformations and Their Applications, Academic Press, New York, NY, USA, 1982.

[3] R. Hirota, "Exact solution of the korteweg-de vries equation for multiple Collisions of solitons," Physical Review Letters, vol. 27, no. 18, pp. 1192-1194, 1971.

[4] M. Song and Z. Liu, "Periodic wave solutions and their limits for the generalized KP-BBM equation," Journal of Applied Mathematics, vol. 2012, Article ID 363879, 25 pages, 2012.

[5] X. Wu, W. Rui, and X. Hong, "Exact traveling wave solutions of explicit type, implicit type, and parametric type for $K(m, n)$ equation," Journal of Applied Mathematics, vol. 2012, Article ID 236875, 23 pages, 2012.

[6] S. Liu, Z. Fu, S. Liu, and Q. Zhao, "Jacobi elliptic function expansion method and periodic wave solutions of nonlinear wave equations," Physics Letters A, vol. 289, no. 1-2, pp. 69-74, 2001.

[7] Z. I. A. Al-Muhiameed and E. A.-B. Abdel-Salam, "Generalized Jacobi elliptic function solution to a class of nonlinear Schrodinger-type equations," Mathematical Problems in Engineering, vol. 2011, Article ID 575679, 11 pages, 2011.

[8] K. A. Gepreel and A. R. Shehata, "Jacobi elliptic solutions for nonlinear differential difference equations in mathematical physics," Journal of Applied Mathematics, vol. 2012, Article ID 710375, 15 pages, 2012.

[9] N. A. Kudryashov, "Exact solutions of the generalized Kuramoto-Sivashinsky equation," Physics Letters A, vol. 147, no. 5-6, pp. 287-291, 1990.

[10] A. A. Soliman and H. A. Abdo, "New exact solutions of nonlinear variants of the RLW, and PHI-four and Boussinesq equations based on modified extended direct algebraic method," International Journal of Nonlinear Science, vol. 7, no. 3, pp. 274-282, 2009.

[11] J.-H. He, "Homotopy perturbation method with an auxiliary term," Abstract and Applied Analysis, vol. 2012, Article ID 857612, 7 pages, 2012.

[12] F. A. Godinez, M. A. Escobedo, and M. Navarrete, "Homotopy analysis method for the Rayleigh equation governing the radial dynamics of a multielectron bubble," Journal of Applied Mathematics, vol. 2012, Article ID 591058, 20 pages, 2012.

[13] J.-H. He and X.-H. Wu, "Exp-function method for nonlinear wave equations," Chaos, Solitons $\mathcal{E}$ Fractals, vol. 30, no. 3, pp. 700-708, 2006.

[14] H. Naher, F. A. Abdullah, and M. Ali Akbar, "New traveling wave solutions of the higher dimensional nonlinear partial differential equation by the exp-function method," Journal of Applied Mathematics, vol. 2012, Article ID 575387, 14 pages, 2012.

[15] S. T. Mohyud-Din, M. A. Noor, and K. I. Noor, "Exp-function method for traveling wave solutions of modified Zakharov-Kuznetsov equation," Journal of King Saud University, vol. 22, no. 4, pp. 213-216, 2010.

[16] H. Naher, F. A. Abdullah, and M. A. Akbar, "The exp-function method for new exact solutions of the nonlinear partial differential equations," International Journal of the Physical Sciences, vol. 6, no. 29, pp. 6706-6716, 2011.

[17] J.-M. Kim and C. Chun, "New exact solutions to the KdV-Burgers-Kuramoto equation with the Expfunction method," Abstract and Applied Analysis, vol. 2012, Article ID 892420, 10 pages, 2012.

[18] A. M. Wazwaz, "A new (2+1)-dimensional Korteweg-de-Vries equation and its extension to a new (3+1)-dimensional Kadomtsev-Petviashvili equation," Physica Scripta, vol. 84, no. 3, Article ID 035010, 2011.

[19] Y. Liu, H. Li, J. Wang, and W. Gao, "A new positive definite expanded mixed finite element method for parabolic integrodifferential equations," Journal of Applied Mathematics, vol. 2012, Article ID 391372, 24 pages, 2012.

[20] H. Ni, L.-X. Tian, and Y. Hong-Xing, "On the positive almost periodic solutions of a class of nonlinear Lotka-Volterra type system with feedback control," Journal of Applied Mathematics, vol. 2012, Article ID 135075, 15 pages, 2012.

[21] J. He, "Variational iteration method for delay differential equations," Communications in Nonlinear Science and Numerical Simulation, vol. 2, no. 4, pp. 235-236, 1997.

[22] Q. Liu and R. Xu, "Periodic solutions of a Cohen-Grossberg-type BAM neural networks with distributed delays and impulses," Journal of Applied Mathematics, vol. 2012, Article ID 643418, 17 pages, 2012.

[23] M. Noor, K. Noor, A. Waheed, and E. A. Al-Said, "An efficient method for solving system of thirdorder nonlinear boundary value problems," Mathematical Problems in Engineering, vol. 2011, Article ID 250184, 14 pages, 2011. 
[24] Y. Li and C. Wang, "Three positive periodic solutions to nonlinear neutral functional differential equations with parameters on variable time scales," Journal of Applied Mathematics, vol. 2012, Article ID 516476, 28 pages, 2012.

[25] Z. I. A. Al-Muhiameed and E. A.-B. Abdel-Salam, "Generalized hyperbolic function solution to a class of nonlinear Schrodinger-type equations," Journal of Applied Mathematics, vol. 2012, Article ID 265348, 15 pages, 2012.

[26] A. H. Bhrawy, A. S. Alofi, and S. I. El-Soubhy, "An extension of the Legendre-Galerkin method for solving sixth-order differential equations with variable polynomial coefficients," Mathematical Problems in Engineering, vol. 2012, Article ID 896575, 13 pages, 2012.

[27] A. Biswas and E. Zerrad, "1-soliton solution of the Zakharov-Kuznetsov equation with dual-power law nonlinearity," Communications in Nonlinear Science and Numerical Simulation, vol. 14, no. 9-10, pp. 3574-3577, 2009.

[28] J. F. Alzaidy, "Extended mapping method and its applications to nonlinear evolution equations," Journal of Applied Mathematics, vol. 2012, Article ID 597983, 14 pages, 2012.

[29] M. Wang, X. Li, and J. Zhang, "The $\left(G^{\prime} / G\right)$-expansion method and travelling wave solutions of nonlinear evolution equations in mathematical physics," Physics Letters A, vol. 372, no. 4, pp. 417-423, 2008.

[30] J. Feng, W. Li, and Q. Wan, “Using $\left(G^{\prime} / G\right)$-expansion method to seek the traveling wave solution of Kolmogorov-Petrovskii-Piskunov equation," Applied Mathematics and Computation, vol. 217, no. 12, pp. 5860-5865, 2011.

[31] H. Naher, F. A. Abdullah, and M. A. Akbar, "The $\left(G^{\prime} / G\right)$-expansion method for abundant traveling wave solutions of Caudrey-Dodd-Gibbon equation," Mathematical Problems in Engineering, vol. 2011, Article ID 218216, 11 pages, 2011.

[32] E. M. E. Zayed and S. Al-Joudi, "Applications of an extended ( $\left.G^{\prime} / G\right)$-expansion method to find exact solutions of nonlinear PDEs in mathematical physics," Mathematical Problems in Engineering, vol. 2010, Article ID 768573, 19 pages, 2010.

[33] K. A. Gepreel, "Exact solutions for nonlinear PDEs with the variable coefficients in mathematical physics," Journal of Information and Computing Science, vol. 6, no. 1, pp. 003-014, 2011.

[34] R. Abazari and R. Abazari, "Hyperbolic, trigonometric and rational function solutions of HirotaRamani Equation via $\left(G^{\prime} / G\right)$-expansion method," Mathematical Problems in Engineering, vol. 2011, Article ID 424801, 11 pages, 2011.

[35] T. Ozis and . Aslan, "Application of the $\left(G^{\prime} / G\right)$-expansion method to Kawahara type equations using symbolic computation," Applied Mathematics and Computation, vol. 216, no. 8, pp. 2360-2365, 2010.

[36] H. Naher, F. A. Abdullah, and M. A. Akbar, "New traveling wave solutions of the higher dimensional nonlinear evolution equation by the improved $\left(G^{\prime} / G\right)$-expansion method," World Applied Sciences Journal, vol. 16, no. 1, pp. 11-21, 2012.

[37] H. Naher and F. A. Abdullah, "Some new traveling wave solutions of the nonlinear reaction diffusion equation by using the improved $\left(G^{\prime} / G\right)$-expansion method," Mathematical Problems in Engineering, vol. 2012, Article ID 871724, 17 pages, 2012.

[38] H. Naher and F. A. Abdullah, "Some new solutions of the combined KdV-MKdV equation by using the improved $\left(G^{\prime} / G\right)$-expansion method," World Applied Sciences Journal, vol. 16, no. 11, pp. 15591570, 2012.

[39] H. Naher and F. A. Abdullah, "The improved ( $\left.G^{\prime} / G\right)$-expansion method for the (2+1)-dimensional Modified Zakharov-Kuznetsov equation," Journal of Applied Mathematics, vol. 2012, Article ID 438928, 20 pages, 2012.

[40] S.-D. Zhu, "The generalizing Riccati equation mapping method in non-linear evolution equation: application to $(2+1)$-dimensional Boiti-Leon-Pempinelle equation," Chaos, Solitons and Fractals, vol. 37, no. 5, pp. 1335-1342, 2008.

[41] A. Bekir and A. C. Cevikel, "The tanh-coth method combined with the Riccati equation for solving nonlinear coupled equation in mathematical physics," Journal of King Saud University, vol. 23, pp. 127-132, 2011.

[42] S. Guo, L. Mei, Y. Zhou, and C. Li, "The extended Riccati equation mapping method for variablecoefficient diffusion-reaction and mKdV equations," Applied Mathematics and Computation, vol. 217, no. 13, pp. 6264-6272, 2011.

[43] B. Li, Y. Chen, H. Xuan, and H. Zhang, "Generalized Riccati equation expansion method and its application to the $(3+1)$-dimensional Jumbo-Miwa equation," Applied Mathematics and Computation, vol. 152, no. 2, pp. 581-595, 2004. 
[44] A. Salas, "Some exact solutions for the Caudrey-Dodd-Gibbon equation," Mathematical Physics. In press, http://128.84.158.119/abs/0805.2969v2.

[45] H. Naher and F. A. Abdullah, "The modified Benjamin-Bona-Mahony equation via the extended generalized Riccati equation mapping method," Applied Mathematical Sciences, vol. 6, no. 111, pp. 5495-5512, 2012.

[46] M. Khalfallah, "New exact traveling wave solutions of the $(2+1)$ dimensional Zakharov-Kuznetsov (ZK) equation," Analele stiintifice ale Universitatii Ovidius Constanta, vol. 15, no. 2, pp. 35-44, 2007.

[47] A. Bekir, "Application of the $\left(G^{\prime} / G\right)$-expansion method for nonlinear evolution equations," Physics Letters A, vol. 372, no. 19, pp. 3400-3406, 2008. 




Advances in

Operations Research

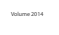

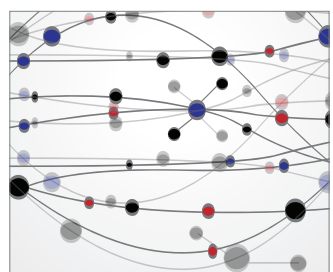

\section{The Scientific} World Journal


International Journal of

Mathematics and

Mathematical

Sciences
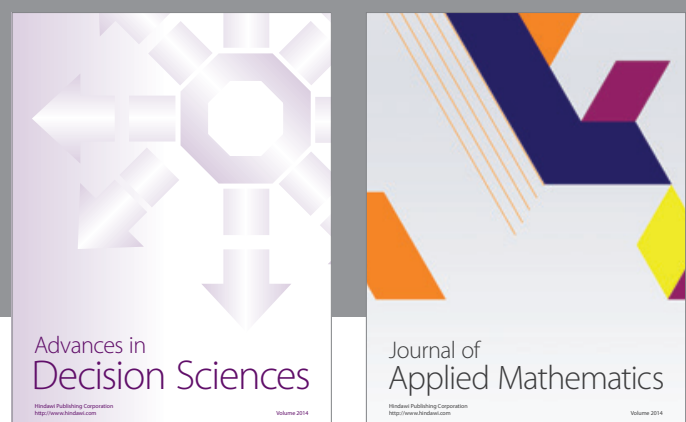

Journal of

Applied Mathematics
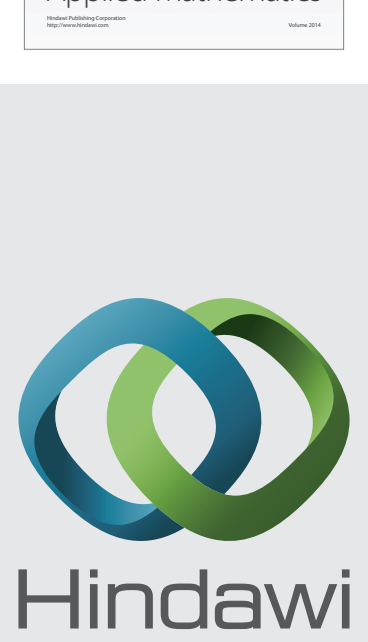

Submit your manuscripts at http://www.hindawi.com
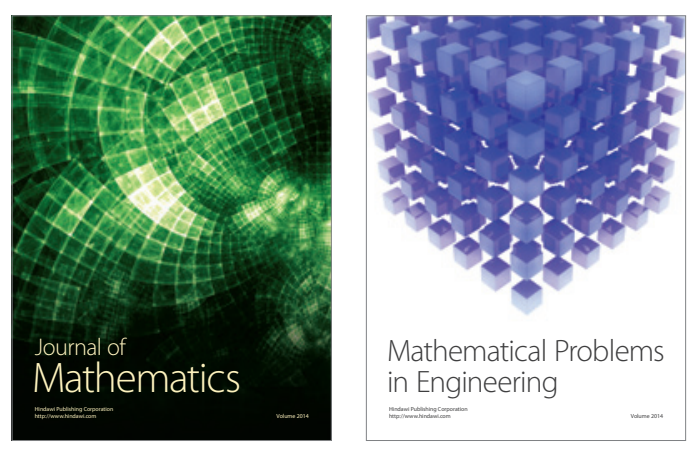

Mathematical Problems in Engineering
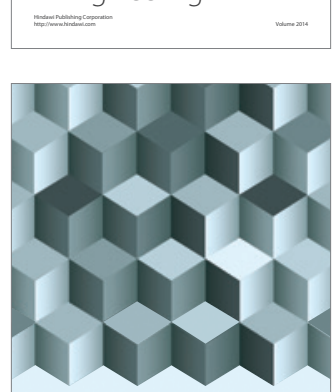

Journal of

Function Spaces
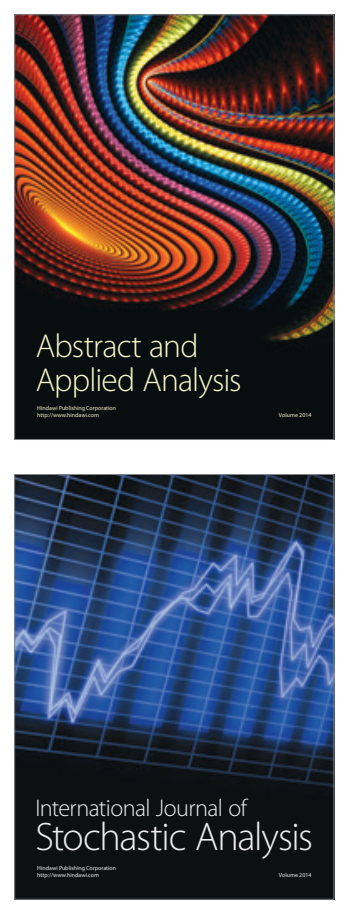

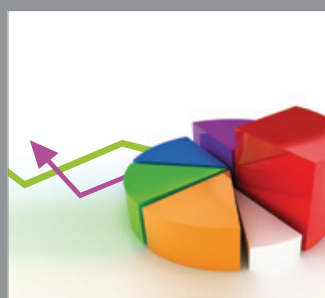

ournal of

Probability and Statistics

Promensencen
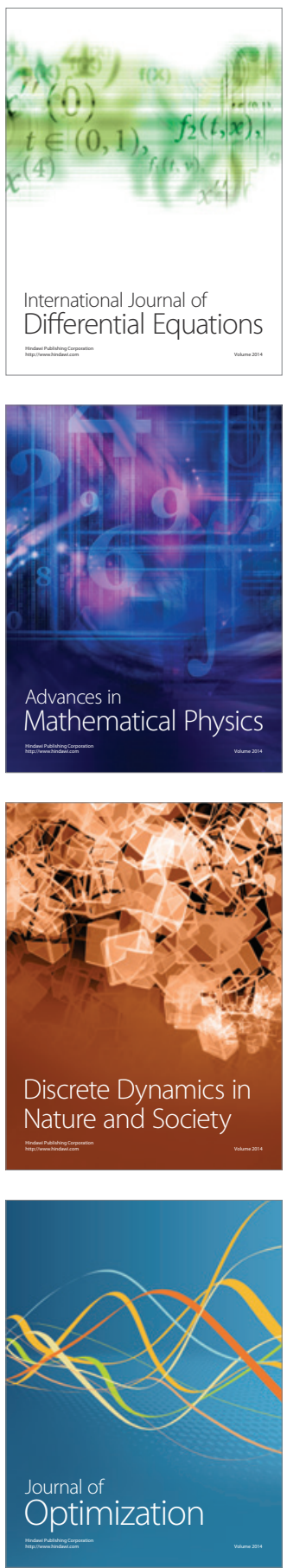